\title{
Handheld electrochemical device for the determination of the strength of garlic
}

Kamonwad Ngamchuea, Philip Hurst, Christopher Batchelor-McAuley, Richard G.

Compton*

*corresponding author: Richard G. Compton, Department of Chemistry, Physical \& Theoretical Chemistry Laboratory, University of Oxford, South Parks Road, Oxford, OX1 3QZ, United Kingdom

Email: richard.compton@ chem.ox.ac.uk. Tel: +44(0)1865275957 Fax: +44(0)1865275410

\begin{abstract}
A handheld electrochemical sensor has been demonstrated for the quantification of the strength of garlic. The device is based on the enhanced voltammetric response in the presence of organosulfur compounds extracted from garlic. Convenient and disposable platinum screen-printed electrodes are employed. All measurements and data analyses are performed within the device. A linear response of the voltammetric peak current enhancement as a function of garlic concentration was observed, indicating the ability of the device to be applied to garlic samples of any strength. Importantly, this portable sensor can be used by non-scientifically skilled personnel and does not require expensive laboratory equipment. It is thus suitable for application in the food industry.
\end{abstract}

\section{Keywords}

Garlic; screen printed electrode; food quality; electrochemical instrumentation; bromination; organosulfur compounds 


\section{Introduction}

Garlic belongs to the genus Allium which is well-known for its antibiotic activity $(1,2)$, pungency and strong odour. These properties stem from their organosulfur components such as disulfides (R-S-S-R') and sulfoxides (R-S $\left.(=\mathrm{O})-\mathrm{R}^{\prime}\right)$. (3) Upon cell damage, the sulfoxides are biologically converted to thiosulfinates $\left(\mathrm{R}-\mathrm{S}(=\mathrm{O})-\mathrm{S}-\mathrm{R}^{\prime}\right)$ and it is this family of compounds that are responsible for the pungent flavour of garlic. (4) The painful and burning sensation of garlic is induced by thiosulfinates from the alliums activating the pain receptor and triggering inflammation. (3)

Thanks to its unique odour and properties, garlic in both raw and processed forms is widely used as a cooking ingredient. However, long term storage of the bulbs prior to processing is known to alter the sulfoxide composition. $(5,6)$ Moreover, different cooking methods (7), sub-species, sources and harvest time of garlic results in a variability of the flavour and strength of garlic. (8-10)

For consistency of the quality of the final consumer products, the strength of garlic and the amount required must be reviewed frequently on a batch-to-batch basis before they can be used in industrial-scale food production. At present, the 'strength' of garlic is assessed in the food industry via organoleptic testing. However, in the preparation of ready-made meals, cooking sauces, garlic powder, medicinal supplements and many other garlic containing products, large quantities of garlic have to be tested. This demands a more reliable, quicker and accurate method than taste testing.

Scientifically, various techniques are available for the analysis of organosulfur concentration such as mass spectrometry (11), high performance liquid chromatography (12-14), gas chromatography $(15,16)$, capillary electrophoresis $(17)$ and electrochemical methods $(18$, 19). To date, electrochemical bromination (19) is the most appropriate method for industrial usage due to its relatively low cost, short experimental time and ease of use for nonscientifically trained operators. The electrochemical method uses the bromide/bromine redox system as the homogeneous redox mediator at a platinum macro-electrode. Organosulfur is extracted from garlic using the less toxic solvent, ethyl acetate. The method has been proved to be able to cover the whole range of garlic strength and the results showed an excellent correlation with organoleptic tests. (19) 
In this paper, we develop a small, portable device to carry out the electrochemical bromination at point-of-use. The device can perform cyclic voltammetry measurements using a custom-made potentiostat. Data analysis is done within the device hence no prior knowledge of electrochemistry or data processing is required. Disposable screen-printed electrodes are employed instead of conventional macro-electrodes to discard the step of electrode preparation. The novelty of this paper lies in the design of the device, which below we term the 'Garlic Meter.' The investigation of the differences between screen-printed electrodes and macro-electrodes, and the origins underlying them is also evidenced. We show how limitations and difficulties in the use of screen-printed platinum electrodes can be overcome and demonstrate the capability of this Garlic Meter to authentic chopped garlic samples of different concentrations.

\section{Materials and Methods}

\subsection{Materials}

Sodium bromide (M\&D Laboratory Chemicals, >99\%), sodium perchlorate monohydrate (Sigma-Aldrich, 98\%) and ethyl acetate (Sigma-Aldrich, $\geq 99.7 \%$ ) were used as received. Garlic samples were provided by Beacon Foods Ltd. All solutions were prepared using deionised water (Millipore) with a resistivity of $18.2 \mathrm{M} \Omega \mathrm{cm}$ at $25^{\circ} \mathrm{C}$. Measurements were conducted using disposable screen-printed electrodes (Dropsens ref. 550, $4 \mathrm{~mm}$ diameter (20)) consisting of platinum working electrode, platinum counter electrode and silver pseudo reference electrode.

\subsection{Design of the Garlic Meter}

The electronic circuit of the device is outlined schematically in Figure 1a. The device includes i) a three-electrode potentiostat built from three OP196 operational amplifiers to conduct electrochemical measurement; ii) PIC microcontroller programmed in PICBASIC PRO Compiler to operate the device; iii) 12-bit digital-to-analog converter (DAC) to supply the voltage set by microcontroller to the amplifier; iv) 12-bit analog-to-digital converter (ADC) to deliver the signal obtained from the amplifier to the microcontroller; v) a switch labelled as 'START' to turn on the device and commence the electrochemical measurement; vi) a switch labelled as 'CONTINUE' to start the second measurement (discussed further below); vii) a voltage regulator to maintain a constant voltage level; viii) two $12 \mathrm{~V}$ DC 
relays; ix) 10k ohm resistor labelled as ' $\mathrm{R} 1$ ' in the diagram allows the flow of currents up to the maximum of ca. $130 \mu \mathrm{A} ; \mathrm{x}$ ) The lower resistor $\mathrm{R} 2$ is put in the circuit during the conditioning phase, this allows larger currents through the working electrode to allow enough charge to pass for surface oxidation of the platinum. In addition, the second relay reconfigures the inputs to allow more voltage between the counter and reference electrodes during the oxidizing activation phase. The normal three-electrode configuration is used for the reducing activation phase as the potentials are less. The device is powered by four $1.5 \mathrm{~V}$ AAA batteries. The values of voltage applied throughout the measurement are summarized in Figure $1 \mathrm{~b}$ as a function of time.

Figure 1: a) Schematic diagram of the electronic circuit of the Garlic Meter with the picture of the meter showing at the top right corner; $b)$ the voltage $\left(V_{\mathrm{WE}}\right)$ applied as a function of time

The Garlic Meter is designed to perform three major functions: electrode activation, two cyclic voltammetry measurements and data analysis; described next.

\section{a) Electrode Activation}

Pre-treatment of the platinum screen-printed electrodes (SPEs) is required prior to performing electrochemical measurements, as will be evidenced later. This is done by holding the potential at $1.7 \mathrm{~V}$ for $2 \mathrm{~s}$ to electrochemically oxidize platinum and impurities on the 
electrode surface, followed by the application of $-0.1 \mathrm{~V}$ for $5 \mathrm{~s}$. At this potential, all platinum oxides formed during the electrode activation at $1.7 \mathrm{~V}$ would be reduced back to platinum as reported by Vetter et al. (21) that complete reduction of platinum oxide occurs at $0.6 \mathrm{~V}$ vs SHE ( $\sim 0.85 \mathrm{~V}$ vs Ag). The system is then left at $0.0 \mathrm{~V}$ for $5 \mathrm{~s}$ prior to the cyclic voltammetric measurement.

\section{b) Cyclic Voltammetry}

The voltage at the working electrode is varied linearly as a function of time, relative to the reference electrode where the potential is scanned from $0 \mathrm{~V}$ to $1.2 \mathrm{~V}$ and back to $0 \mathrm{~V}$ at a scan rate of $100 \mathrm{mV} \mathrm{s}^{-1}$. The Garlic Meter first runs a cyclic voltammogram for the control solution (no organosulfur is present), and then the same voltammogram is repeated in the presence of the garlic sample. Both scans are required for the quantification of garlic strength as described in the following section.

\section{c) Data Acquisition and Analysis}

Due to the partial onset of the oxidation of the metal surface, it is likely that the currents at the anodic end of the measurement may be higher than the diffusion-limited peak currents of the redox couple under study. The Garlic Meter is therefore designed to run the scan up to $1.2 \mathrm{~V}$ to obtain the appropriate range for cyclic voltammogram, but only allows the detection of the peak current up to $1.1 \mathrm{~V}$; refer to Figure $2 \mathrm{a}$.

At the end of the measurements, the raw data of the current as a function of time (or voltage) can be obtained by connecting the Garlic Meter to a personal computer via USB connection. For industrial use and hence for the use by non-scientifically skilled personnel, analysis is performed internally by the garlic meter. Here, the screen is programmed to display the peak currents of the first and second scans as well as the baseline currents of both scans taken at $0.7 \mathrm{~V}$ as a first review point. The screen shows these values of peak currents for three seconds, then advances to the next page displaying the results of baseline corrected peak currents for both scans. The result of final data analysis which is the normalized current enhancement $\left(i_{\mathrm{p}}-i_{\mathrm{p}, 0} / i_{\mathrm{p}, 0}\right)$ calculated by a microcontroller is shown at the bottom line of this screen. This current enhancement quantifies the amount of organosulfur in the garlic sample. The need for this baseline subtraction will be discussed in Section 3.2. 
Figure 2: a) Diagram showing the voltage ranges of cyclic voltammetry measurement and peak detection; b) screen displays where $I$ is the current measured and $i$ is baseline corrected current. Subscripts $\mathrm{p}, \mathrm{b}$ and 0 denote peak, baseline and control respectively.

\subsection{Procedures}

Organosulfur was extracted from a crushed garlic sample $(0.2 \mathrm{~g}-1.0 \mathrm{~g})$ by adding $2 \mathrm{~mL}$ of ethyl acetate to the centrifuge tube containing a known amount of garlic. The tube was vortexed for $1 \mathrm{~min}$ and subsequently centrifuged at $4400 \mathrm{rpm}$ for $6 \mathrm{~min}$. The resulting ethyl acetate supernatant was added to the sodium bromide aqueous solution containing an excess amount of sodium perchlorate salts to give the final concentration of ethyl acetate, sodium bromide and sodium perchlorate of $5 \% \mathrm{v} / \mathrm{v}, 1.0 \mathrm{mM}$ and $0.1 \mathrm{M}$ respectively.

For the electrochemical measurements, a $5 \% \mathrm{v} / \mathrm{v}$ solution of pure ethyl acetate in sodium bromide solution $\left(1.0 \mathrm{mM} \mathrm{NaBr}\right.$ and $\left.0.1 \mathrm{M} \mathrm{NaClO}_{4}\right)$ was used as a control. A $50 \mu \mathrm{L}$ aliquot of this solution was dropped onto the platinum screen-printed electrode. This amount is adequate to cover all three electrodes on the chip. Cyclic voltammetry was then conducted by pressing the 'START' button.

Second, a $50 \mu \mathrm{L}$ aliquot of the solution containing the garlic extract was dropped onto the same SPE as the control after washing carefully with deionized water and subsequently dried. The second measurement was performed by pressing 'CONTINUE' on the device. 


\section{Results and Discussion}

\subsection{Electrode Activation}

Ethyl acetate is the solvent used for the extraction of organosulfur from garlic. It has been reported that the oxidation of sodium bromide in the presence of $5 \% \mathrm{v} / \mathrm{v}$ ethyl acetate gives a well-defined voltammogram with a relatively small peak-to-peak separation of ca. $88 \mathrm{mV}$ (at $100 \mathrm{mV} \mathrm{s}^{-1}$ ) on a platinum macro-electrode. (19)

First, the oxidation of bromide was studied on the screen-printed electrodes to compare the results with those obtained from a conventional macro-electrode. Preliminary experiments on the screen-printed electrodes were performed using $\mu$ Autolab Type III potentiostat. The CVs of the sodium bromide solution $\left(1.0 \mathrm{mM} \mathrm{NaBr}, 0.1 \mathrm{M} \mathrm{NaClO}_{4}, 5 \%\right.$ ethyl acetate) at a scan rate of $100 \mathrm{mV} \mathrm{s}^{-1}$ recorded at a non-activated Pt SPE showed much smaller peak current in the forward scan and the backward peak was absent; refer to Figure 3. This suggests the decrease in electrode activity of the Pt SPE as compared with the corresponding macroelectrode. As SPEs cannot be polished, the formation of an oxide film, the adsorption of impurities on the electrode surface and the difference in microstructures from diverse manufacturing procedures are potential factors accounting for the observed lack of electrode activity.

The above result demonstrates that a suitable method is required to achieve a higher surface activity of the Pt SPEs. Electrochemical activation was chosen in this case so that both electrode activation and the voltammetric measurement can be performed consecutively using a single device. For optimum oxidation of the platinum surface, at least $4.5 \mathrm{mC}$ of charge has to be passed through the electrode. This value of charge was estimated from chronoamperometry of Pt SPE in fully supported $1.0 \mathrm{mM} \mathrm{NaBr}$ (aq) at $1.7 \mathrm{~V}$ for $2 \mathrm{~s}$ (not shown). All of the platinum oxide formed must then be reduced back to platinum prior to recording the $\mathrm{CV}$. The system is next left at $0.0 \mathrm{~V}$ for another $5 \mathrm{~s}$ for the diffusion layer at the electrode to relax back to its initial state. These optimized activating conditions are summarized in Section 2.2a. A well-defined cyclic voltammogram similar to that obtained from a macro-electrode was subsequently obtained when the electrode had been activated as described, as recorded using the commercial potentiostat (Figure 3, blue line). This change in the voltammetric response evidences that the electrochemical pre-treatment of the SPEs 
successfully remove the impurities and help to increase the activity of electrode towards electron transfer in a similar manner to polishing of macro-electrodes.

For the electrode activation using the Garlic Meter, the 10k ohm resistor (R1, Figure 1a) used in the circuit does not allow enough charge to be transferred within the intended time interval $(2 \mathrm{~s})$, and so a well-defined voltammogram cannot be obtained from this conventional potentiostat set-up (single gain). Consequently, the Garlic Meter incorporates an additional relay within the handheld device to switch the current path to the milliamps current range allowing at least $4.5 \mathrm{mC}$ to pass through in $2 \mathrm{~s}$. The time interval of electrode activation $(2 \mathrm{~s})$ was chosen to be the shortest possible to minimize the operation time and optimize the process allowed by the $6 \mathrm{~V}$ supplied by four AAA $1.5 \mathrm{~V}$ batteries. Figure 3 (green line) shows that with appropriate pre-treatment of the electrode, a well-defined cyclic voltammogram can be obtained using the Garlic Meter.

Figure 3: Cyclic voltammograms showing current densities of fully supported $1.0 \mathrm{mM} \mathrm{NaBr}$ (aq) utilising i) Pt macro-electrode (polished with alumina powder) / $\mu$ Autolab Type III potentiostat (black, dash); ii) Pt SPEs / $\mu$ Autolab Type III potentiostat with (blue) and without (red) electrode activation; iii) Pt SPEs / Garlic Meter with electrode activation (green) 


\subsection{Detection of Organosulfur by Electrochemical Bromination}

In the previous section, we have obtained the appropriate conditions for the oxidation of bromide using Pt SPEs. The changes in voltammetric response and especially the peak currents in the presence of organosulfur will be investigated next. Cyclic voltammetry of the control sample $\left(1.0 \mathrm{mM} \mathrm{NaBr}, 0.1 \mathrm{M} \mathrm{NaClO}_{4}, 5 \%\right.$ ethyl acetate) was first carried out at a scan rate of $100 \mathrm{mV} \mathrm{s}^{-1}$. Upon the addition of the garlic extract, bromine formed by the electro-oxidation of bromide ion reacts with the disulfides and sulfoxides present to form thiosulfonates and reform bromide. (22) This electrocatalytic oxidation reaction (an EC' mechanism) (23) leads to redox cycling of the bromide and hence results in an enhanced voltammetric peak current. According to the electrocatalytic cycle summarized in Figure 4, the magnitude of current enhancement is proportional to the concentration of disulfides and sulfoxides, and hence directly related to the strength of garlic. $(18,19)$

Figure 4: Schematic diagram of an electrocatalytic cycle with bromide/bromine redox couple as a heterogeneous mediator in the conversion of disulfide or sulfoxide (RSSR) to thiosulfonates $\left(\mathrm{RSO}_{3} \mathrm{H}\right)$

Although the use of the bromide/bromine redox mediator at a Pt macro-electrode for the quantification of garlic strength has been shown to be successful, measurements on Pt SPEs impose major problems which require further adjustments towards the development of the Garlic Meter. The cyclic voltammogram of sodium bromide solution in the presence of 5\% $\mathrm{v} / \mathrm{v}$ ethyl acetate supernatant containing extracted compounds from chopped garlic sample exhibits a pre-wave at $\sim 0.5 \mathrm{~V}$. The presence of the pre-wave potentially causes error in the quantification of organosulfur concentrations as the magnitude of the peak current (and hence current enhancement) is altered. This is evidenced by the fact that the linear extrapolation of the calibration plot (current enhancement vs. mass of garlic) did not pass through zero- 
intercept. In other words, extrapolation of current enhancement to zero mass (i.e. no organosulfur present) results in non-zero value causing systematic error to the measurement of garlic strength. An alternative approach to quantification was thus sought.

Studies at varied scan rates showed that both the currents of pre-wave and the bromide oxidation wave increase with scan rate. The ratio of the bromide oxidative peak currents $\left(I_{\mathrm{p}}\right)$ to the current of the pre-wave $\left(I_{\mathrm{b}}\right)$ decreases with scan rate; refer to inlay in Figure 5 . One might infer that measurement at slow scan rates would minimize the systematic error caused by this pre-wave; however, this is not the case for Pt SPEs. At slow scan rate of $25 \mathrm{mV} \mathrm{s}{ }^{-1}$, reproducible CVs cannot be obtained due to the interference of oxidation of other organic molecules or impurities present. Consequently, relatively fast scan rates are required to minimize the influence of the side reactions. $\mathrm{CV}$ studies at slower scan rate of $10 \mathrm{mV} \mathrm{s}{ }^{-1}$ revealed the nature of this pre-wave. In accordance with previous report by Wopschall et al. (24), a wave occurring at a potential less positive than the formal potential of the redox couple of interest likely result from the strong adsorption of the reaction product at the electrode surface. The amount of charge transfer integrated from the pre-wave is ca. $4.3 \mu \mathrm{C}$ which is equivalent to $1.1 \times 10^{14}$ molecules $\mathrm{cm}^{-2}$. This value is comparable in magnitude to the value of $1.5 \times 10^{15}$ atoms $\mathrm{cm}^{-2}$ suggested by Bard and Faulkner (25) to represent the value of monolayer adsorption on $\mathrm{Au}$ (111) surface.

Figure 5: $\mathrm{CVs}$ of $1.0 \mathrm{mM} \mathrm{NaBr}, 0.1 \mathrm{M} \mathrm{NaClO} 4,5 \% \mathrm{v} / \mathrm{v}$ ethyl acetate supernatant from the extraction of organosulfur from $0.6 \mathrm{~g}$ of garlic sample at the scan rates of $25 \mathrm{mV} \mathrm{s}^{-1}$ (red), 50 $\mathrm{mV} \mathrm{s}^{-1}$ (blue), $100 \mathrm{mV} \mathrm{s}^{-1}$ (pink), $200 \mathrm{mV} \mathrm{s}^{-1}$ (green) and $400 \mathrm{mV} \mathrm{s}^{-1}$ (dark blue); the inlay shows the ratio of peak current to baseline current (measured at $0.7 \mathrm{~V}$ ) at the different scan rates. 
At fast scan rate of $100 \mathrm{mV} \mathrm{s}^{-1}$, the pre-wave is a plateau; in contrast to the well-defined peak obtained at slow scan rates. It can therefore be treated in term of data analysis as a baseline. The Garlic Meter measures the baseline current $\left(I_{b}\right)$ at $0.7 \mathrm{~V}$. Its value is subtracted from the voltammetric peak current $\left(I_{\mathrm{p}}\right)$ to yield the baseline corrected peak current $\left(i_{\mathrm{p}}\right)$. Series of experiments were performed for varied masses of garlic used in the extraction step to obtain a calibration plot. The current enhancements calculated from baseline corrected currents give a straight line as a function of mass of garlic with $\mathrm{R}^{2}$ value of 0.99 passing through zerointercept; shown in Figure 6. The precision of the measurements is within $\pm 5 \%$ error of the mean values measured for each garlic strength. The errors are at least an order of magnitude smaller than the difference in the voltammetric signal recorded between two garlic samples differing by $0.2 \mathrm{~g}$ in mass. Furthermore, this error is less than $7 \%$ of the anticipated range of the industrially used garlic samples.

The results were analysed within the Garlic Meter. The linear calibration plot thus verified three conclusions. First, the programmed microcontroller can successfully carry out i) cyclic voltammetry measurements; ii) baseline subtraction and iii) calculation of the current enhancement. Second, the Garlic Meter can determine the strength of garlic for both 'weak' and 'strong' garlic as resembled by measurements at low mass $(0.2 \mathrm{~g})$ and high mass $(1.0 \mathrm{~g})$ respectively. Third, the peak currents range from ca. $-40 \mu \mathrm{A}$ to $100 \mu \mathrm{A}$, suggesting that the use of $10 \mathrm{k}$ ohm resistor is appropriate for this system $(-130 \mu \mathrm{A}$ to $130 \mu \mathrm{A})$. For uses in other systems, this resistor R1 (refer to Figure 1a) can be easily exchanged to appropriate size. The above results have thus demonstrated that the strength of garlic has been successfully quantified using the Garlic Meter.

Figure 6: $\mathrm{CVs}$ of $1.0 \mathrm{mM} \mathrm{NaBr}, 0.1 \mathrm{M} \mathrm{NaClO} 4,5 \% \mathrm{v} / \mathrm{v}$ ethyl acetate supernatant from the extraction of organosulfur from different masses of garlic samples at the scan rate of $100 \mathrm{mV} \mathrm{s}^{-1}$; $0 \mathrm{~g}$ (black), $0.2 \mathrm{~g}$ (red), $0.4 \mathrm{~g}$ (blue), $0.6 \mathrm{~g}$ (pink), $0.8 \mathrm{~g}$ (green), $1.0 \mathrm{~g}$ (dark blue); the inlay shows the calibration plot of current enhancement $\left(i_{\mathrm{p}}-i_{\mathrm{p}, 0} / i_{\mathrm{p}, 0}\right)$ as a function of mass of garlic. 


\section{Conclusion}

A handheld device has been developed to determine the strength of garlic sensitively and quantitatively. It offers the advantages of using disposable screen-printed electrodes and as a result also reduces the amount of garlic required for each measurement. This provides a good basis for industrial batch-to-batch quality control. It requires neither expensive laboratory equipment nor specific scientific training, and therefore is suitable for direct application in the food industry. It has been proved that the Garlic Meter covers the whole range of garlic strength. With this inexpensive electroanalytical tool, we can eliminate the variability of results from organoleptic testing.

\section{Acknowledgements}

We would like to thank Beacon Foods (Brecon, UK) for providing the garlic samples. KN receives funding from the Royal Thai government (DPST scholarship). RGC acknowledges funding from the ERC Grant Agreement [no. 320403].

\section{References}

1. P. Karuppiah, S. Rajaram, Antibacterial effect of Allium sativum cloves and Zingiber officinale rhizomes against multiple-drug resistant clinical pathogens. Asian Pac. J. Trop. Biomed. 2, 597-601 (2012)10.1016/s2221-1691(12)60104-x).

2. D. S. Arora, J. Kaur, Antimicrobial activity of spices. Int. J. Antimicrob. Agents 12, 257-262 (1999)10.1016/s0924-8579(99)00074-6).

3. E. Block, Garlic and Other Alliums: The Lore and the Science. (RSC Pub., 2010).

4. M. H. Brodnitz, J. V. Pascale, Vandersl.L, Flavor Components of Garlic Extract. J. Agric. Food. Chem. 19, 273-\& (1971)10.1021/Jf60174a007).

5. M. Ichikawa, N. Ide, K. Ono, Changes in organosulfur compounds in garlic cloves during storage. J. Agric. Food. Chem. 54, 4849-4854 (2006); published online EpubJun 28 (10.1021/jf060083o).

6. G. M. Volk, K. E. Rotindo, W. Lyons, Low-temperature storage of garlic for spring planting. HortScience 39, 571-573 (2004)

7. T. H. Yu, C. M. Wu, C. T. Ho, Volatile compounds of deep-oil fried, microwaveheated and oven-baked garlic slices. J. Agric. Food. Chem. 41, 800-805 (1993)10.1021/jf00029a023).

8. J. Hornickova, R. Kubec, K. Cejpek, J. Velisek, J. Ovesna, H. Stavelikova, Profiles of S-Alk(en)ylcysteine Sulfoxides in Various Garlic Genotypes. Czech J. Food Sci. 28, 298-308 (2010).

9. J. Hornickova, R. Kubec, J. Velisek, K. Cejpek, J. Ovesna, H. Stavelikova, Changes of S-alk(en)ylcysteine Sulfoxide Levels During the Growth of Different Garlic Morphotypes. Czech J. Food Sci. 29, 373-381 (2011). 
10. E. Block, S. Naganathan, D. Putman, S. H. Zhao, Allium chemistry: HPLC analysis of thiosulfinates from onion, garlic, wild garlic (ramsoms), leek, scallion, shallot, elephant (great-headed) garlic, chive, and Chinese chive. Uniquely high allyl to methyl ratios in some garlic samples. J. Agric. Food. Chem. 40, 2418-2430 (1992)10.1021/jf00024a017).

11. S. N. Lee, N. S. Kim, D. S. Lee, Comparative study of extraction techniques for determination of garlic flavor components by gas chromatography-mass spectrometry. Anal. Bioanal. Chem. 377, 749-756 (2003); published online EpubOct (10.1007/s00216-003-2163-z).

12. S. J. Ziegler, O. Sticher, HPLC of S-Alk(en)yl-L-cysteine Derivatives in Garlic including Quantitative Determination of (+)-S-Allyl-L-cysteine Sulfoxide (Alliin). Planta Med. 55, 372-378 (1989); published online EpubAug (10.1055/s-2006962031).

13. M. Ichikawa, N. Ide, J. Yoshida, H. Yamaguchi, K. Ono, Determination of seven organosulfur compounds in garlic by high-performance liquid chromatography. $J$. Agric. Food. Chem. 54, 1535-1540 (2006); published online EpubMar 8 (10.1021/jf051742k).

14. Y. Ueda, H. Kawajiri, N. Miyamura, R. Miyajima, Content of Some SulfurContaining Components and Free Amino-Acids in Various Strains of Garlic. J. Jpn. Soc. Food Sci. Technol. 38, 429-434 (1991).

15. R. Kubec, M. Svobodová, J. Velíšek, Distribution ofS-Alk(en)ylcysteine Sulfoxides in SomeAlliumSpecies. Identification of a New Flavor Precursor: S-Ethylcysteine Sulfoxide (Ethiin). J. Agric. Food. Chem. 48, 428-433 (2000)10.1021/jf990938f).

16. R. Kubec, M. Svobodova, J. Velisek, Gas chromatographic determination of Salk(en)ylcysteine sulfoxides. J. Chromatogr. A 862, 85-94 (1999); published online EpubNov 5 (10.1016/S0021-9673(99)00902-4).

17. R. Kubec, E. Dadakova, Quantitative determination of S-alk(en)ylcysteine-S-oxides by micellar electrokinetic capillary chromatography. J. Chromatogr. A 1212, 154-157 (2008); published online EpubNov 28 (10.1016/j.chroma.2008.10.024).

18. B. C. Martindale, L. Aldous, N. V. Rees, R. G. Compton, Towards the electrochemical quantification of the strength of garlic. The Analyst 136, 128-133 (2011); published online EpubJan 7 (10.1039/c0an00706d).

19. E. M. Hall, K. Tschulik, C. Batchelor-McAuley, R. G. Compton, Electrochemical bromination of organosulfur containing species for the determination of the strength of garlic (A. sativum). Food Chem. 199, 817-821 (2016)10.1016/j.foodchem.2015.12.086).

20. DropSens Screen-Printed Platinum Electrodes (Ref. 550). Available at: http://www.dropsens.com/en/pdfs_productos/new_brochures/550.pdf. (Accessed: 6 January 2016).

21. K. J. Vetter, J. W. Schultze, The kinetics of the electrochemical formation and reduction of monomolecular oxide layers on platinum in $0.5 \mathrm{M} \mathrm{H} 2 \mathrm{SO} 4 . J$. Electroanal. Chem. Interfacial Electrochem. 34, 131-139 (1972)10.1016/s00220728(72)80509-6).

22. S. Siggia, R. L. Edsberg, Determination of Alkyl Sulfides and Disulfides. Anal. Chem. 20, 938-939 (1948)10.1021/ac60022a021).

23. R. G. Compton, C. E. Banks, Understanding Voltammetry. (Imperial College Press, ed. 2nd, 2011), pp. 444.

24. R. H. Wopschall, I. Shain, Effects of adsorption of electroactive species in stationary electrode polarography. Anal. Chem. 39, 1514-1527 (1967)10.1021/ac50156a018). 
25. A. J. Bard, L. R. Faulkner, Electrochemical Methods: Fundamentals and Applications. (Wiley, 2000).

\section{Biographies}

Kamonwad Ngamchuea is a D.Phil Student in the Compton Group. She is working on the electrochemical detection of organosulfur molecules. She graduated with a master's degree in Chemistry at the University of Oxford in 2015.

Philip Hurst is a technician in the electronics workshop in the Physical Chemistry Laboratory, the University of Oxford, since 1991.

Chris Batchelor-McAuley studied and completed (2012) his D.Phil under the guidance of Professor Compton at the University of Oxford. His current work is focused on the fundamental and electrochemical properties of nanoparticles. To date he has published over 90 papers, has a h-index of 18 and is a co-author of the book 'Understanding Voltammetry: Problems and Solutions'

Richard G. Compton is Aldrichian Praelector, and Professor at the University of Oxford (UK). He has published in excess of 1400 papers $(h=86)$, numerous patents and 7 books. He is the Editor-in-Chief and the Founding Editor of the journal Electrochemistry Communications (Elsevier). 